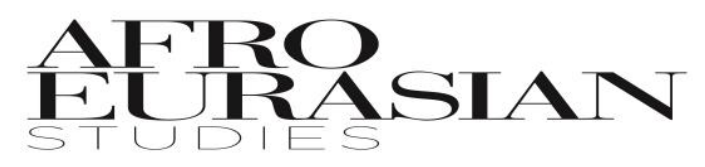

[Afes], 2018, 7 (2): 240-264

Cross-Cultural Examination of Emotional Expressivity, Beliefs About Emotions, and Depression of University Students

\title{
Zihniye OKRAY
}

Doç. Dr., Lefke Avrupa Üniversitesi, Fen Adebiyat Fakültesi Psikoloji Bölümü Assoc. Prof. Dr., Psychology Department, Arts and Science Faculty, European University of Lefke, zokray@eul.edu.tr, Orcid ID: 0000-0002-9117-4991 Sultan OKUMUŞOĞLU

Yrd. Doç. Dr., Lefke Avrupa Üniversitesi, Fen Adebiyat Fakültesi Psikoloji Bölümü Asst. Prof. Dr., Psychology Department, Arts and Science Faculty, European University of Lefkessehitoglu@eul.edu.tr, Orcid ID: 0000-0001-6155-2325 Cemaliye DIREKTÖR

Dr., Lefke Avrupa Üniversitesi, Fen Adebiyat Fakültesi Psikoloji Bölümü /

Dr., Psychology Department, Arts and Science Faculty, European University of Lefke cdirektor@eul.edu.tr, Orcid ID: 0000-0002-6055-2224

Angelika Hüseyinzade ŞIMŞEK

Y.L.., Çağ Üniversitesi, Fen Edebiyat Fakültesi, Psikoloji Bölümü MSc., Psychology Department, Arts and Science Faculty, Çağ University anjelikasimsek@cag.edu.tr, Orcid ID: 0000-0003-4297-1780

\section{Article Information}

Article Types : Research Article

Received : :09.11.2018

Accepted : :29.12.2018

Published : :31.12.2018

Pub Date Season: Fall

Cite as: OKRAY, Z. OKUMUŞOĞLU, S, DİREKTÖR, C, HÜSEYİNZADE ŞİMŞEK, A. (2018). Cross-Cultural Examination of Emotional Expressivity, Beliefs About Emotions, and Depression of University Students. Afro Eurasian Studies, 7 (1), 240-264. Retrieved from http://dergipark.gov.tr/afes/issue/39788/471071

Plagiarism: This article has been reviewed by at least two referees and scanned via a plagiarism software. http://www.dergipark.gov.tr/afes

Copyright (C) Published by MUSIAD- Sutluce Mah. Imrahor Cad. No:28 34445 Beyoglu Istanbul- Turkey Phone: +90 - 212 - 3950000 Fax: +90 - 212 - 3950001 Email: aestudies@musiad.org.tr 


\title{
Cross-Cultural Examination of Emotional Expressivity, Beliefs About Emotions, and Depression of University Students
}

\begin{abstract}
This study investigates the cross-cultural differences between emotional expressivity, beliefs about emotions, and depression levels among university students from different continents, namely, Africa and Asia. Participants ( $n=114 ; n=88$, Africa and $n=26$, Asia) were English speaking students of European University of Lefke studying at different faculties (Social Sciences, Engineering, Health, and Agriculture). They completed Beliefs about Emotions Scale (BES), Berkeley Expressivity Questionnaire (BEQ), and The Center for Epidemiologic Studies Depression Scale (CESD-R). Findings suggested that Asian students' CESD-R sadness and suicidal ideation subscale scores, as well as BEQ positive expressivity scores, have been found higher than the scores of African students. Female
\end{abstract}


students differed with significantly higher scores than males of CESD-R guilt subscale and BEQ impulse strength subscale score. Further, a significant difference between CESD-R scores according to class was obtained as well, junior students had higher scores than senior students. CESD-R total scores indicate that Health Faculty students' scores are higher than the scores of Engineering Faculty students. As a result, Asian students found to be more prone to sadness than African students.

Keywords: African, Asian, Depression, Emotional Expressivity, Beliefs About Emotions, University Students.

\section{Introduction}

The core difference between human beings and other species is the ability to express emotions. Expressing emotions is an important factor that affects individuals' psychological wellbeing because it is again the central part of interpersonal relations and communication (Johnson, 1997). Individual's disparate in terms of emotion expression degree is conceptualized as emotional expressivity (Kring, Smith, \& Neale, 1994). Despite some suggestions pointing out contradictory results (Leising, Grande, \& Faber, 2010) regarding the relationship of emotional experience and 
expressivity with psychopathology, i.e., revealing a change in psychopathological symptoms and no change in emotional expressivity following psychotherapy, the general claim is different. According to plenty of health professionals, emotional experience and expressivity are related to psychopathology (Greenberg, \& Pascual-Leone, 2006; McCullough, 2001). The same association was also pointed out by many theoreticians with different perspectives (Ainsworth, Bell, \& Stayton, 1974; Freud, 1957; Jenkins and Oatley, 1996; Mann, 1996). Therefore, many psychotherapists target improvement of their clients' emotional expressivity (Leising, Müller, \& Hahn, 2007).

\section{Emotional Expressivity}

Studies which relates emotional expressivity with well-being suggest that emotional expressivity has an important role not only in psychopathologies like depression (Sloan, Strauss, \& Wisner, 2001) but also in interpersonal interactions (King, 1993) through possible misunderstandings or lack of communication.

Theoretical contributions to the recognition of emotions can be summarized as follows. From the behavioral approach, emotional expressivity is based on learning, learning emotions, in particular, influenced by early life experiences. To establish a relationship with others, it is necessary for 
infants to learn to recognize emotions and express them (Karabuschenko, Ivashchenko, Sungurova \& Mihailovna Hvorova, 2016, Russell, Bacharowski \& Fernandez-Dols, 2003).

Cognitive Theory proposes that individuals' cognitions, automatic thoughts, assumptions, and beliefs are important with regards to the development and maintenance of psychopathologies (Beck, 1967; Beck, Emery, \& Greenberg, 1985). Therefore, having dysfunctional acceptability beliefs in respect of emotional experience and expressivity would have an important association with the development and maintenance of various psychopathologies such as depression (Ferster, 1973), social anxiety (Clark \& Wells, 1995), and eating disorders (Corstorphine, 2006).

People who have dysfunctional beliefs about the possible negative consequences of emotional experience and expressivity get benefits from the restructuring of these dysfunctional cognitions. Hence, it is obvious that individuals' beliefs about possible negative consequences of emotional expressivity have a strong impact on the way of emotion regulation used by them (Juretić \& Zivcić-Bećirevic, 2013), therefore, have an impact on their mood. Dysfunctional beliefs would lead to the engagement in safety behaviors, and safety behaviors would promote disapproval from others (Rachman, Grüter-Andrew, \& Shafran, 2000; Wells et al., 
1995), which will cause other negative consequences as depressive mood or maintenance of pathologies.

Cross-cultural studies show that especially emotions of happiness, sadness, surprise, disgust, anger, and fear are universally recognized emotions but there are always some variations in their expression and recognition that are dependent on individual's culture which they grow in (Ekman et al., 1987; Shioiri et al., 1999). The recognition of emotion across cultures is similar, while the way in which emotions might be represented or labeled appears to be more culture-specific (DeKlerk, Dada \& Alant, 2014). In that matter, effects of culture, thus, cross-cultural differences concerning the emotional expressions and symptoms were emphasized in some studies (Lehti, Johansson, Bengs, Danielsson, Hammarstrom, 2010). Effects of ethnicity in terms of emotional expressivity and mood ( $\mathrm{Lu} \&$ Wang, 2012) were also pointed out in the literature. Iwata and Buka (2002) conducted a survey to investigate cross-cultural/ethnic comparison among university students from East Asia as well as North and South America and found out that East Asian students have low positive affect and high depressive scores, Native Americans have negative symptoms and low positive affect, and South Americans appear to have low depressive symptoms. Young, Fang, and Zisook (2010) studied depression among Asian-American and Caucasian 
undergraduate students and discovered that AsianAmericans showed higher levels of depression and KoreanAmerican students are at risk. Although Burgin et al. (2012), Khan et al. (2008), and Gross et al. (2000) used university students in their researches they did not mention and crosscultural results about emotional expressivity in their papers. Akın (2012) studied the relationship between emotional expressivity and loneliness of university students and unearthed that loneliness was predicted negatively by positive expressivity, negative expressivity, and impulse strength. In another research, Akın et al. (2012) investigated the relationship between emotional expressivity and submissive behavior, and the results showed that submissive behavior is negatively associated with emotional expressivity.

The sample of this study consists of volunteer students from two different continents, Africa and Asia. The aim of this study is to examine the differences between emotional expressivity, beliefs about emotions, and depression levels of university students who were born in different continents. The secondary aim of this study is to examine the differences in emotional expressivity, beliefs about emotions, and depression levels of students with regards to their gender, relationship status, class, and faculty.

\section{Research Method}




\section{Participants}

The data was collected in the European University of Lefke among English speaking students who were enrolled in compulsory courses -History and Foreign Language. In this frame, questionnaires were distributed and collected from these target classes. After the elimination of incomplete surveys, the final sample consisted of 114 students. The mean age of the sample was calculated as $21.26 \pm 3.23$ with the range of 16 to 36 . The distributions of the socio-demographical variables of the sample were shown in Table 1.

Table1. Distribution of sociodemographic variables $(\mathrm{N}=114)$

Variable

\section{Gender}

Female

44

38.6

Male

70

61.4

Faculty

Social Sciences

Engineering 56

Health

Agriculture

11

9.6

7

6.1

Class 
Junior

Senior

Relationship Status

Single

Living with Partner

3

2.6

Married

5

4.4

Separated

3

\section{Birth Continent}

Africa

\section{Data Collection Tools}

\section{Socio-Demographic Information Form}

Demographic information form was generated by researchers to get information from the participants about their age, gender, birth continent, faculty, class, and relationship status.

\section{The Beliefs about Emotions Scale (BES)}

BES was developed by Rimes and Chalder (2010) to measure beliefs about experiencing and expressing negative thoughts and feelings. Each item is answered on a 0-6 scale, in which 0 indicates 'totally disagree' and 6 indicates 'totally agree'. All items are phrased in a positive direction. Scores can range 
from 0 to 72. Internal consistency of the scale was found to be 0.91. The principal component analysis of BES was calculated and only one factor was extracted, which accounted for the $50.1 \%$ of the variance (Rimes and Chalder, 2010).

\section{Berkeley Expressivity Questionnaire (BEQ)}

BEQ was developed by Gross and John (1997) to evaluate three facets of emotional expressivity, i.e., negative expressivity, positive expressivity, and impulse strength. Internal consistency scores were calculated as 0.86 for the total $\mathrm{BEQ}$, and $0.70,0.70$, and 0.80 for the three subscales negative expressivity, positive expressivity, and impulse strength, respectively. Each item is answered on a 1-7 scale, in which 1 indicates 'strongly disagree' and 7 indicates 'strongly agree'. Items 3, 8, and 9 were reverse coded. From the scale, either a total score can be calculated to measure emotional expressivity or 3 different scores can be calculated to measure negative emotionality, positive emotionality, and impulse strength (Gross and John, 1997).

\section{The Center for Epidemiologic Studies Depression Scale (CESD-R)}

The Center for Epidemiological Studies Depression Scale (CES-D) developed by Radloff (1977) is a short self-report scale for screening purposes and used in scientific studies for the assessment of depressive symptoms in the general 
population. CESD-R is the revised version of it in 2004 by Eaton and others (2004). The 20 items in CESD-R scale measure symptoms of depression in nine different groups as defined by the American Psychiatric Association Diagnostic and Statistical Manual, fifth edition (Eaton, Muntaner, Smith, Tien, Ybarra, 2004).

\section{Findings}

1. Findings related to the differences between emotional expressivity, beliefs about emotions, and depression according to the continent of birth.

Statistically significant mean differences were found between Asian and African students from subscales of CESD-R sadness and suicidal ideation scores as well as BEQ positive expressivity subscale scores $(\mathrm{p}<0.05)$. Inspection of the two group means indicates that average CESD-R sadness and suicidal ideation subscale scores, as well as BEQ positive expressivity score of Asian students are higher than the scores of African students.

No statistically significant mean differences were found between Asian and African students in CESD-R total score and its subscales, BES total score, BEQ negative expressivity, and BEQ impulse strength scores ( $p>0.05)$.

2. Findings related to the differences between emotional expressivity, beliefs about emotions, and depression according to gender. 
Statistically significant mean differences were found between female and male students from CESD-R guilt score and BEQ impulse strength score $(\mathrm{p}<0.05)$. Inspection of the two group means shows that average CESD-R guilt score and BEQ impulse strength score of female students are higher than that of male students.

No statistically significant mean differences of female and male students on CESD-R total score and its subscales, BES total score, BEQ negative expressivity, and BEQ positive expressivity scores were found ( $p>0.05)$.

3. Findings related to the differences between emotional expressivity, beliefs about emotions, and depression according to class.

Statistically significant mean differences were found between junior and senior students from of CESD-R total score as well as sadness, appetite, sleep, thinking, tiredness, and agitation subscale scores $(\mathrm{p}<0.05)$. Inspection of the two group means indicates that average CESD-R scores of junior students are higher than the scores of senior students.

No statistically significant mean differences were found between junior and senior students on BES total score and BEQ subscale scores ( $p>0.05)$.

4. Findings related to the correlation between emotional expressivity, beliefs about emotions, and depression with relationship status. 
According to ANOVA results, statistically significant differences were found among BEQ negative expressivity ( $F$ $(3.110)=3.242, \mathrm{p}=0.025, \mathrm{p}<0.05)$ and BEQ positive expressivity $(\mathrm{F}(3.110)=3.220, \mathrm{p}=0.026, \mathrm{p}<0.05)$. Inspection of the two group means of BEQ negative expressivity scores shows that the mean scores of single students are higher than the scores of separated students. Furthermore, the BEQ positive expressivity scores indicate that the students who are living with their partners have higher scores than married students.

According to ANOVA results, no statistically significant differences were found among the four groups of marital status, i.e., single, separated, married, and living with the partner in CESD-R total score and subscales, BES and BEQ impulse strength subscale ( $p>0.05)$.

5. Findings related to the correlation between emotional expressivity, beliefs about emotions, and depression with faculty.

According to ANOVA results, statistically significant differences were found among CESD-R total score $(\mathrm{F}(3.110)=$ 3.613, $\mathrm{p}=0.016, \mathrm{p}<0.05)$ as well as sleep $(\mathrm{F}(3.110)=3.583$, $\mathrm{p}=0.016, \mathrm{p}<0.05)$, thinking $(\mathrm{F}(3.110)=2.915, \mathrm{p}=0.038, \mathrm{p}<0.05)$, and agitation $(\mathrm{F}(3.110)=4.358, \mathrm{p}=0.006, \mathrm{p}<0.05)$ subscales and BES total score $(\mathrm{F}(3.110)=3.314, \mathrm{p}=0.023, \mathrm{p}<0.05)$. Inspection of the two group means of CESD-R total scores indicates that the mean scores of Health Faculty students are higher than that of 
Engineering Faculty students. Also, inspection of sleep and thinking subscales showed similar results as Health Faculty students sleep and thinking subscale scores are higher than Engineering Faculty students'. Agitation subscale scores of CESD-R showed that Social Science Faculty students' scores are higher than Engineering Faculty students' scores. Moreover, the BES total scores indicate that the students who are in Social Sciences Faculty have higher scores than Health Faculty students.

According to ANOVA results, no statistically significant differences were found among the four faculty groups, namely Social Sciences Faculty, Engineering Faculty, Health Faculty, and Agriculture Faculty in BEQ subscales and some of the CESD-R subscales ( $p>0.05$ ).

6. Findings related to the correlation between emotional expressivity, beliefs about emotions, and depression.

The relationship between BES, BEQ, and CESD-R was calculated with the Pearson product-moment correlation coefficient. According to the results, CESD-R total score was positively correlated with BEQ positive expressivity $(r=0.231$, $p=0.013, p<0.05)$ and BEQ impulse strength $(r=0.200, p=0.032$, $\mathrm{p}<0.05)$. In addition, BES total score was positively correlated with BEQ negative expressivity $(r=0.235, p=0.012, p<0.05)$.

\section{Discussion}


As can be seen in results no differences have been found between Asian and African students regarding CESD-R total score and its subscales, BES total score, BEQ negative expressivity, and BEQ impulse strength scores. Asian students' CESD-R sadness and suicidal ideation subscale scores, as well as BEQ positive expressivity scores, have been found higher than the scores of African students. On the other hand, correlational analyses revealed that CESD-R total score was positively correlated with BEQ positive expressivity and BEQ impulse strength. Also, BES total scores were positively correlated with BEQ negative expressivity. It seems that dysfunctional beliefs regarding the expression of emotions tended to rise together with the expression of emotions including anger and nervousness more frequently and depressive tendencies tended to rise together with positive expressivity and impulse strength. This means dysfunctional beliefs regarding the expression of emotions tended to rise together with depressive mood. It was pointed out in the literature since inter- and intra-personal processes are influenced by emotional tendencies, individual differences with respect to these tendencies are important (Gross \& John, 1995) and mentioned results are consistent with this claim. Effects of ethnicity concerning emotional expressivity and mood (Lu \& Wang, 2012) were also presented in the literature. 
Some suggest that since culture could be related with the expressions and symptoms of some psychopathologies like depression, a probable misdiagnosis in cross-cultural assessment should be taken into consideration (Lehti, Johansson, Bengs, Danielsson, Hammarstrom, 2010).

Female students differed with significantly higher means than males regarding the scores of CESD-R guilt subscale and BEQ impulse strength subscale. This finding can be accepted as parallel with the literature (Kring, Smith, \& Neale, 1994) which points out that women are emotionally more expressive than men. According to Kring et al. (1994), women are more emotionally expressive regarding the expression of emotions such as shame, sadness, and fear. Despite the claim that more emotional expressivity is related to less anxiety and guilt feelings and more happiness, it is obvious that the nature of the expressed feelings is crucial. For example, if the expressed feeling is impulsive anger expression, it will probably deteriorate the individual's social relationships, physical health, and psychological well-being (Kring, Smith, \& Neale, 1994). This finding is also consistent with the other result of the present study, which underlines that depressive tendencies were tended to arise together with impulse strength.

Average CESD-R scores as well as sadness, appetite, sleep, thinking, tiredness, and agitation subscale scores of junior 
students are found higher than the scores of senior students. It makes sense since junior students are still in the orientation period to their new environment and this points out the need to provide support for junior students in their adaptation during the orientation period.

Regarding the BEQ negative expressivity mean scores, single students are found to have higher scores than the scores of separated students. Additionally, analyses regarding the BEQ positive expressivity scores indicate that the students who are living with their partners have higher scores than the married ones. The way of emotional expressivity of individuals seems different between single and separated students. Frequent expression of anger or nervousness is included in BEQ positive expressivity subscale. It was found that depression tended to rise together with BEQ positive expressivity, which reminds that emotional expressivity not only has an important role with respect to psychopathologies like depression (Sloan, Strauss, \& Wisner, 2001) but also in interpersonal interactions (King, 1993) through probable misunderstandings or lack of communication.

Health Faculty students' CESD-R total mean scores as well as sleep and thinking subscale scores are found higher than the scores of Engineering Faculty students. Social Science Faculty students' agitation subscale scores of CESD-R and BES total score are found higher than Engineering Faculty students' 
scores. Future studies regarding other differences among faculties could be helpful to understand related factors as having their different subcultures with different display rules or even perhaps as having different curriculums.

\section{Conclusion}

Studies which relates emotional expressivity with well-being suggest that emotional expressivity not only has an important role in psychopathologies like depression (Sloan, Strauss, \& Wisner, 2001) but also in interpersonal interactions (King, 1993) through probable misunderstandings or lack of communication.

People who have dysfunctional beliefs about possible negative consequences for emotional experience and expressivity get benefits from the restructuring of these dysfunctional cognitions.

Cross-cultural researches showed that especially emotions of happiness, sadness, surprise, disgust, anger, and fear are universally recognized emotions but there are always some differences in terms expression and recognition that are dependent on individual's culture which they grow in (Ekman et al., 1987; Shioiri et al., 1999).

The aim of this study was to examine the cross-cultural differences between emotional expressivity, beliefs about emotions, and depression levels of university students. 
Relationship of culture -which is important in terms of assessment and treatment too- regarding the expression of emotions and mood as well as the relationship of cognition with psychological well-being were pointed out by results. Besides, the necessity to provide support for international junior students during the orientation period has been emphasized.

\section{Kaynakça / References}

Ainsworth, M.D.S., Bell, S.M., \& Stayton, D.J. (1974). Infantmother attachment and social development: "Socialization" as a product of reciprocal responsiveness to signals. In P.M. Richards (Ed.) The integration of a child into a social world. Cambridge, UK: Cambridge University Press, 99-135.

Akın, A. (2012). Emotional Expressivity and Loneliness in Religious and Moral Studies Education Students, TOJCE: The Online Journal of Counselling and Education, 1(3): 31-40.

Akın, A., Satıcı, S.A., \& Kayış, A.R. (2012). Emotional Expressivity and Submissive Behavior, Journal of Education and Instructional Studies. 2(1): 1-6.

Beck, A.T. (1967). Depression: Clinical, experimental and theoritical aspects. New York: Harper and Row Press. 
Beck, A.T., Emery, G., \& Greenberg, R.L. (1985). Anxiety disorders and phobias: A cognitive perspective. New York: Basic Books.

Burgin, C. J., Brown, L. H., Royal, A., Silvia, P. J., BarrantesVidal, N., \& Kwapil, T. R. (2012). Being with others and feeling happy: Emotional expressivity in everyday life. Personality and Individual Differences, 53(3), 185-190.

Clark, D.M., \& Wells, A. (1995). A cognitive model of social phobia. In: Heimberg RG,Liebowitz, M.R., Hope, D.A., \& Schneier, F.R. editors. Social Phobia: Diagnosis, assessment and treatment (pp 69-93). New York: Guilford Press.

Corstorphine, E. (2006). Cognitive-emotional-behavioural therapy for the eating disorders: Working with beliefs about emotions. Europian Eating Disorders Review.14, 448-461.

DeKlerk, H.M, Dada, S., \& Alant, E. (2014). Children's identification of graphic symbols representing four basic emotions: Comparison of Afrikaans-speaking and Sepedispeaking children. Journal of Communication Disorders, 52:1-15.

Eaton, W.W., Muntaner, C., Smith, C., Tien, A., \& Ybarra, M. (2004). Center for Epidemiologic Studies Depression Scale: Review and revision (CESD and CESD-R). In: Maruish ME, Ed. The Use of Psychological Testing for Treatment Planning 
and Outcomes Assessment. 3rd ed. Mahwah, NJ: Lawrence Erlbaum; 363-377.

Ekman, P., Friesen, W. V., O'Sullivan, M., Chan, A., Diacoyanni-Tarlatzis, I., \& Heider, K. (1987). Universals and cultural differences in the judgments of facial expression of emotion. Journal of Personality and Social Psychology, 53(4), 712-717.

Ferster, C.B., (1973). A functional analysis of depression. American Psychologist. 28, 857- 870.

Freud, S. (1957). Repression. In J. Strachey (Ed. and Trans.), The standard edition of the complete psychological works of Sigmund Freud (Vol. 14, pp. 143-158). London: Hogarth Press (Original work published 1915).

Greenberg, L.S., \& Pascual-Leone, A. (2006). Emotion in psychotherapy: A practice-friendly review. Journal of Clinical Psychology, 62, 611-630.

Gross, J.J., \& John, O.P. (1995). Facets of emotional expressivity: Three self-report factors and their correlates. Personality and Individual Differences, 19, 555-568. http://doi.org/cks

Gross, J.J., \& John, O.P., (1997). Revealing feelings: Facets of emotional expressivity in self-reports, peer ratings, and 
behavior. Journal of Personality and Social Psychology, 72:435-448.

Gross, J.J., John, O.P., \& Richards, J.M.(2000). The Dissociation of emotion Expression from Emotion Experience: A Personality Perspective, Personality and Social Psychology Bulletin, 26(6):712-726.

Iwata, N., \& Buka, S.(2002). Race/ethnicity and depressive symptoms: a cross-cultural/ethnic comparison among university students in East Asia, North and South America, Social Science \& Medicine, 55:2243-2252.

Jenkins, J.M., \& Oatley, K. (1996). Emotional episodes and emotionality through the lifespan. In C. Magai \& S.H. McFadden (Eds.), Handbook of emotion, adult development and aging (pp. 421-441). San Diego, CA: Academic Press.

Johnson, D. W. (1997). Reaching out: Interpersonal effectiveness and self-actualization. Boston: Allyn \& Bacon.

Juretić, J., \& Zivcić-Bećirevic, I. (2013). Social Anxiety, Beliefs About Expressing Emotions and Experiencing Positive Emotions, New Insights into Anxiety Disorders, Dr. Federico Durbano (Ed.),InTech, DOI: 10.5772/55110. Available from: https://www.intechopen.com/books/new-insights-intoanxiety- disorders/ social-anxiety-beliefs-about-expressingemotions-and-experiencing-positive-emotions. 
Karabuschenko, N.B., Ivashchenko A.V., Sungurova, N.L. \& Hvorova,E.M.(2016). Emotion Recognition in Different Cultures, Indian Journal of Science and Technology, 9(48); 117.

Kahn, J.H., Barr, L.K., \&Schneider, J.W.(2008). Individual Differences in Emotion Expression: Hierarchical Structure andRelations with Psychological Distress.Faculty Publications - Psychology. 2.

http://ir.library.illinoisstate.edu/fppsych/2.

King, L. A. (1993). Emotional expression, ambivalence over expression, and marital satisfaction. Journal of Social and Personal Relationships, 10, 601-607.

Kring, A.M., Smith, D.A., \& Neale, J. M. (1994). Individual differences in dispositional expressiveness: Development and validation of the Emotional Expressivity Scale. Journal of Personality and Social Psychology, 66, 934-949. http://doi.org/ckw

Lehti, A.H., Johansson, E.E., Bengs, C., Danielsson, U., \& Hammarstrom, A. (2010). 'The western gaze' -an analysis of medical research publications concerning the expressions of depression, focusing on ethnicity and gender. Health Care Women International. 31,100-112. 
Leising, D., Grande, T., \& Faber, R. (2010). A longitudinal study of emotional experience, expressivity, and psychopathology in psychotherapy inpatients and psychologically healthy persons. Journal of Clinical Psychology, 66 (10), 1027-1043.

Leising, D., Müller, J., \& Hahn, C. (2007). An adjective list for assessing emotional expressivity in psychotherapy research. Clinical Psychology \& Psychotherapy, 14, 377-385.

Lu, W., \& Wang, Z. (2012). Emotional expressivity, emotion regulation, and mood in college students: A cross-ethnic study. Social Behavior and Personality, 40(2), 319-330.

Mann, S.J. (1996). Severe paroxysmal hypertension: An autonomic syndrome and its relationship to repressed emotions. Psychosomatics: Journal of Consultation and Liaison Psychiatry, 37, 444-450.

McCullough, L. (2001). Assimilative integration: Short-term dynamic psychotherapy for treating affects phobias. Clinical Psychology-Science and Practice, 8, 82-97.

Rachman, S., Grüter-Andrew, J., \& Shafran, R. (2000). Postevent processing in social anxiety. Behaviour Research and Therapy, 38, 611-617. 
Radloff, L.S. (1977). The CES-D scale: a self-report depression scale for research in the general population. Applied Psychological Measurement, 1,385-401.

Rimes, K.A., \& Chalder, T. (2010). The Beliefs about Emotions Scale: validity, reliability and sensitivity to change. Journal of Psychosomatic Research, 68,285-292.

Russell, J.A., Bacharowsk,J.A., Fernandez-Dols, J.M.(2003) Facial and vocal expression of emotion. Annual review of psychology. 54(1):329-49.

Shioiri, T., Someya, T., Helmeste, D., \& Tang, S. W. (1999). Misinterpretation of facial expression: A cross-cultural study. Psychiatry and Clinical Neurosciences, 53,45-50.

Sloan, D. M., Strauss, M.E., \& Wisner, K.L. (2001). Diminished response to pleasant stimuli by depressed women. Journal of Abnormal Psychology, 110, 488-493.

Wells, A., Clark, D.M., Salkovskis, P., Ludgate, J., Hackmann, A., \& Gelder, M.G. (1995). Social phobia: The role of insituation safety behaviors in maintaining anxiety and negative beliefs. Behavior Therapy, 26, 153-161.

Young, C.B., Fang, D.Z., \& Zisook, S.(2010). Depression in Asian-American and Caucasian undergraduate students, Journal of Affective Disorders, 125: 379-382. 